\title{
Randomized study of doxorubicin-based chemotherapy regimens, with and without sildenafil, with analysis of intermediate cardiac markers
}

Andrew Poklepovic ${ }^{1 *}$ D, Yuesheng $\mathrm{Qu}^{2}$, Molly Dickinson ${ }^{3}$, Michael C. Kontos ${ }^{4}$, Maciej Kmieciak ${ }^{3}$, Elizabeth Schultz ${ }^{4}$, Dipankar Bandopadhyay ${ }^{5}$, Xiaoyan Deng ${ }^{5}$ and Rakesh C. Kukreja ${ }^{4}$

\begin{abstract}
Background: Doxorubicin chemotherapy is used across a range of adult and pediatric malignancies. Cardiac toxicity is common, and dysfunction develops over time in many patients. Biomarkers used for predicting late cardiac dysfunction following doxorubicin exposure have shown promise. Preclinical studies have demonstrated potential cardioprotective effects of sildenafil.

Methods: We sought to confirm the safety of adding sildenafil to doxorubicin-based chemotherapy and assess Nterminal Pro-Brain Natriuretic Peptide (NT-proBNP) and high sensitivity cardiac troponin I (hsTnl) as early markers of anthracycline-induced cardiotoxicity. We randomized 27 patients (ages 31-77, 92.3\% female) receiving doxorubicin chemotherapy using a blocked randomization scheme with randomly permuted block sizes to receive standard chemotherapy alone or with the addition of sildenafil. The study was not blinded. Sildenafil was dosed at $100 \mathrm{mg}$ by mouth daily during therapy; patients took sildenafil three times daily on the day of doxorubicin. Doxorubicin dosing and schedule were dependent on the treatment regimen. Echocardiography was obtained prior to initiation of treatment and routinely thereafter up to 4 years. NT-proBNP and hsTnl were obtained with each cycle before, 1-3 $\mathrm{h}$ after, and $24 \mathrm{~h}$ after doxorubicin.

Results: Fourteen patients were randomized to receive standard doxorubicin chemotherapy alone (14 treated and analyzed), while 13 patients were randomized to the experimental doxorubicin and sildenafil arm (10 treated and analyzed). No toxicity signal was seen with the addition of sildenafil to doxorubicin-based regimens. There was no statistical difference between the treatment arms in relation to the change of mean left ventricular ejection fraction (LVEF) between the first and last evaluation. In both arms, hsTnl levels increased over time; however, elevated hsTnl was not associated with declines in LVEF.
\end{abstract}

Conclusion: Adding sildenafil was safe, but did not offer cardioprotection following doxorubicin treatment. Increases in hsTnl levels were observed over time, but elevations during therapy did not correlate with subsequent decreases in LVEF.

Trial registration: This clinical trial (NCT01375699) was registered at www.clinicaltrials.gov on June 17, 2011.

Keywords: Cardioprotection, Chemotherapy, Biomarker, Clinical trial, Ejection fraction, Strain, Anthracycline, Doxorubicin

\footnotetext{
* Correspondence: andrew.poklepovic@vcuhealth.org

${ }^{1}$ Massey Cancer Center and Department of Internal Medicine, Division of Hematology-Oncology, Virginia Commonwealth University, Box 980070, Richmond, VA 23298, USA

Full list of author information is available at the end of the article
}

(c) The Author(s). 2018 Open Access This article is distributed under the terms of the Creative Commons Attribution 4.0 International License (http://creativecommons.org/licenses/by/4.0/), which permits unrestricted use, distribution, and reproduction in any medium, provided you give appropriate credit to the original author(s) and the source, provide a link to the Creative Commons license, and indicate if changes were made. The Creative Commons Public Domain Dedication waiver (http://creativecommons.org/publicdomain/zero/1.0/) applies to the data made available in this article, unless otherwise stated. 


\section{Introduction}

Drugs within the anthracycline class, including doxorubicin, daunorubicin, and idarubicin, are commonly used in chemotherapeutic regimens to treat various solid and hematological malignancies [1-3]. An important anthracycline-related toxicity that impacts cancer survivors is late-onset cardiac dysfunction. Total lifetime anthracycline exposure is positively correlated with congestive heart failure (CHF), thus limiting anthracyclines as a therapeutic option. Dexrazoxane has been evaluated as a cardio-protectant; however, clinical use has been limited as this agent increases hematologic toxicity, and the impact on clinical effectiveness has been controversial. The FDA has approved the use of dexrazoxane to patients with metastatic breast cancer with $\geq 300 \mathrm{mg} / \mathrm{m}^{2}$ of lifetime doxorubicin exposure [4]. Dexrazoxane is also used for extended doxorubicin dosing in patients with metastatic soft tissue sarcoma in combination with olaratumab [5].

The utilization of cardiac markers to predict or assess the cardiac effect of anthracyclines has shown promise. However, no consensus exists for monitoring or predicting patients at highest risk. Baseline elevated levels of high sensitivity troponin $\mathrm{T}$ have been shown to be associated with declines in LVEF following anthracycline therapy, however it was not associated with CHF in childhood survivors of cancer [6, 7]. Cardiac troponin I (cTnI) has been shown to identify high-risk patients and predict the development of irreversible heart failure in patients receiving trastuzumab [8]. Elevation of cTnI immediately after treatment and at one month after completion have also been shown to correlate with subsequent declines in LVEF [9]. It has also been reported that natriuretic peptides (eg, N-terminal pro-Brain natriuretic peptide [NT-proBNP]) may serve as a biomarker that can identify later chemotherapy-associated cardiotoxicity [10].

Sildenafil is a phosphodiesterase 5 (PDE5) inhibitor commonly used for pulmonary hypertension and erectile dysfunction. Pre-clinical studies suggested that sildenafil may increase the therapeutic index of doxorubicin through anti-tumor effect and protection from cardiotoxicity [11, 12]. In preclinical murine studies, treatment with sildenafil prior to doxorubicin exposure prevented cardiomyocyte apoptosis and myofibrillar disarray, as evidenced by abnormal desmin distribution, lack of Z-line integrity, and abnormal cytoplasmic desmin aggregation in animals treated with doxorubicin alone [13]. Similarly, long-acting PDE5 inhibitor tadalafil improved LVEF in mice and prevented cardiomyocyte apoptosis following doxorubicin treatment through mechanisms involving up-regulation of cGMP and mitochondrial antioxidant enzyme MnSOD without interfering with the chemotherapeutic benefits of doxorubicin [14].

Based on this preclinical data, we hypothesized that the addition of sildenafil could serve as a cardioprotectant during doxorubicin therapy. The study was not designed to evaluate sildenafil as an adjunct anticancer agent, although other studies are underway in that regard (NCT02466802, NCT01817751). We evaluated the safety of concurrent administration of sildenafil during doxorubicin-based chemotherapy. Additionally, we monitored serum biomarkers before, during, and after doxorubicin exposure for each cycle, to identify the impact, if any, that the timing of marker elevation may have on later systolic dysfunction. Patients were followed up with serial echocardiograms including tissue Doppler imaging to evaluate if changes in LVEF or strain were associated with serum biomarker changes.

\section{Methods \\ Patients}

Patients who were undergoing treatment with a chemotherapy regimen with doxorubicin were eligible. Eligibility criteria included: age 18 or older, minimum doxorubicin dose of $40 \mathrm{mg} / \mathrm{m}^{2}$, with infusions not more frequently than weekly. In addition, an Eastern Cooperative Oncology Group (ECOG) performance status of 0,1 , or 2 and life-expectancy greater than one year were required. Single agent doxorubicin and combination chemotherapy were allowed. Patients with prior exposure to doxorubicin were included as long as the last dose was greater than 30 days prior to the current doxorubicin-based regimen and their LVEF was greater than 55\%. Women of childbearing potential and men agreed to use a medically accepted form of birth control for the duration of study and a minimum of six months after the last dose of doxorubicin. Patients with known CHF, LVEF less than 55\%, planned concurrent administration of other investigational agents, swallowing or absorption issues, known hearing loss, hypersensitivity or previous toxicity to study drug, or concurrent chronic nitrate or alpha blocker therapy, were excluded, as were pregnant or nursing women. After six $\mathrm{HER}^{+}$patients were enrolled, an amendment was written to also exclude patients with planned subsequent therapy with a HER2-directed treatment or other anthracyclines besides doxorubicin. Drugs with strong CYP3A4 inhibitors and/or inducers were not concurrently administered during the study with the exception of short course of aprepitant.

\section{Treatment}

Patients were randomly assigned to receive doxorubicin-based chemotherapy plus sildenafil or doxorubicin-based chemotherapy alone. To ensure recruitment balance between the 2 arms and avoid possible risk for selection bias, a blocked randomization scheme with randomly permuted blocks sizes (unknown to the investigators) was conducted by the biostatistician. Sildenafil was initiated prior to doxorubicin and continued for 2 weeks after the last dose of doxorubicin. Sildenafil dosing was a single $100 \mathrm{mg}$ dose daily and three $100 \mathrm{mg}$ doses on the day of doxorubicin treatment. Each dose of doxorubicin 
constituted a cycle. The duration of treatment and cumulative dose of doxorubicin were determined by the regimen chosen for treatment and were at the discretion of the treating physician.

\section{Cardiac function and marker monitoring}

LVEF, a surrogate of cardiac function, was monitored with echocardiography. Echocardiography with tissue Doppler and strain imaging was performed prior to treatment; at 3, 6, and 12 months; and then every 12 months for up to 4 years. For the purposes of monitoring patient safety, a clinically significant deterioration in cardiac function was defined as an absolute 10 percentage point decline in LVEF to below $50 \%$, an absolute LVEF of $45 \%$ or below, or a 20 percentage point decline in LVEF at any level.

Two-dimensional grayscale harmonic images were obtained in the left lateral decubitus position using an iE33 or EPIQ7 ultrasound system (Philips Medical Systems, Andover, MA) equipped with a transthoracic broadband X5-1 matrix transducer (composed of 3040 elements with 1-5 MHz). 2D grayscale harmonic images were acquired in the 3 standard apical views (2-, 3-, and 4-chamber) obtained at frame rates of $\geq 50$ frames/s. Native $2 \mathrm{D}$ images were stored digitally for later off-line analysis.

All echocardiograms were analyzed by a reader blinded to the patient visit number. LVEF was calculated from the apical 4- and 2-chamber views using a modified Simpson biplane method. Myocardial LV deformations were analyzed by speckle tracking using the CMQ software (QLAB 10.3; Philips Medical System, Andover, MA). To assess peak systolic LV longitudinal strain, the endocardial and epicardial borders were traced in the $4 \mathrm{C}, 3 \mathrm{C}$, and $2 \mathrm{C}$ on an end-diastolic frame. The program automatically divided the walls in several segments (LV algorithm based on 17-segment model) and tracked these points on a frame-by-frame basis. When tracking was suboptimal, the borders were readjusted manually.

Pre-treatment levels of cTnI, high-sensitivity troponin I (hsTnI) and NT-proBNP were obtained. High-sensitivity troponin I was determined using a research-phase assay based on LOCI technology and run on a Dimension Vista 1500 System (Siemens Healthcare Diagnostics, Newark, $\mathrm{DE})$. The hsTnI assay has a range of 0.5 to $20,000 \mathrm{pg} / \mathrm{mL}$ and a $10 \%$ coefficient of variation of $3 \mathrm{pg} / \mathrm{mL}$. NT-proBNP was measured on the Dimension Vista 500 Intelligent Laboratory System (Siemens Healthcare Diagnostics). The limit of detection of the NT-proBNP assay is $0.8 \mathrm{pg} / \mathrm{mL}$.

Blood samples were obtained before every dose of doxorubicin, approximately $1-3 \mathrm{~h}$ after each infusion (peak doxorubicin serum concentration), and on day $2(\sim 24 \mathrm{~h})$ following each treatment. These time points were selected to detect both immediate (1-3 h and $24 \mathrm{~h}$ after treatment) and delayed (before the next treatment, typically $2-3$ weeks later) cardiac injury. Serum was separated and stored at $-80{ }^{\circ} \mathrm{C}$ until shipment for batch analysis by Siemens (Newark, DE) for cTnI, hsTnI, and NT-proBNP following the completion of accrual.

\section{Statistical methods}

In addition to the study assignment Arms (doxorubicin/sildenafil; doxorubicin-only), all enrolled subjects were categorized into two Outcome Groups based on an LVEF of less than 50\%, which is considered clinically relevant, at their last study visit. Patients were also categorized based on Strain measurement at the 3-year post-treatment echocardiogram (if 3-year strain data was not available then 4-year strain data was used), comparing those with a less than $-17 \%$ strain measurement (more negative; better cardiac function) to a greater than or equal to $-17 \%$ strain measurement (less negative; worse cardiac function). To assess the longitudinal profiles of hsTnI and NT-proBNP (day 2 sample after each infusion) and their relationship to the explanatory variables such as Arm, LVEF-Group, Strain, Cycle, and their various interactions, separate linear mixed model (LMM) analyses were conducted by using the $\mathrm{AR}(1)$ covariance structure. Furthermore, a logistic regression was used to study association of the other explanatory variables to the dichotomous outcome- LVEF-Group, both for hsTnI and NT-proBNP. The 2-sided significance level was set to $5 \%$ for assessing significance of the estimated parameters. All analyses were performed using the MIXED or LOGISTIC procedure in the SAS (Statistical Analysis System) software (v.9.3).

Adverse events were characterized using Common Terminology Criteria for Adverse Events (CTCAE) version 4. An early stopping rule for the study was developed based on commonly used doxorubicin regimens $[3,15,16]$. Prior information suggested a cumulative rate of Grade 3 or 4 hematologic and non-hematologic toxicity of $60 \%$ or smaller would be acceptable for doxorubicin-based therapies. The stopping rule was designed to ensure that adding sildenafil to doxorubicin did not increase the same type of toxicity to more than $80 \%$. For evaluation of this safety measure, patients underwent clinical and laboratory evaluations. An interim safety analysis examined the difference in LVEF between the two treatment arms using three methods: 1) change in LVEF from the first (baseline) to last visits by the repeated measures analysis of variance (ANOVA); 2) change in LVEF between first and last visits by a pooled t-test; and 3) using levels of LVEF over all visits from baseline up to the 24-month follow-up visit by the repeated measures ANOVA. 


\section{Results}

Of the 31 patients who were assessed for eligibility, 27 were randomized and 24 ultimately received study treatment, as shown in the CONSORT diagram (Fig. 1). Of these 24 patients, most were female $(n=22)$ (Table 1). Ten patients (median age 57, 40-77) received doxorubicin with sildenafil while 14 (median age 50, 31-67) received doxorubicin only. The most common cancer type was breast cancer $(n=20)$. Five patients had metastatic disease at the onset of treatment. The median total dose of doxorubicin was $240 \mathrm{mg} / \mathrm{m}^{2}\left(75-360 \mathrm{mg} / \mathrm{m}^{2}\right)$. Smoking risk was more common in the doxorubicin/sildenafil interventional Arm, and cardiac exposure to radiation (defined as left chest wall radiation) was more common in the doxorubicin alone Arm. Two patients in the experimental Arm and 4 patients in the control Arm underwent treatment with trastuzumab following their course of doxorubicin.

\section{Safety and tolerability}

Sildenafil was generally well tolerated, with well-described sildenafil-associated toxicities (headache, blue-green vision changes, and flushing) reported in a minority of patients (Table 2). Most reported toxicities were Grade 1 or 2 . Two patients with Grade 3 headache required dose reductions of sildenafil. There were no Grade 4 toxicities associated with therapy. The threshold for the early stopping rule was not reached, but accrual to the trial was halted for interim safety analysis after a patient who received sildenafil with doxorubicin developed severe LVEF decline. This patient was on adjuvant trastuzumab at that time, and attribution to therapy was unclear. The $p$-value for

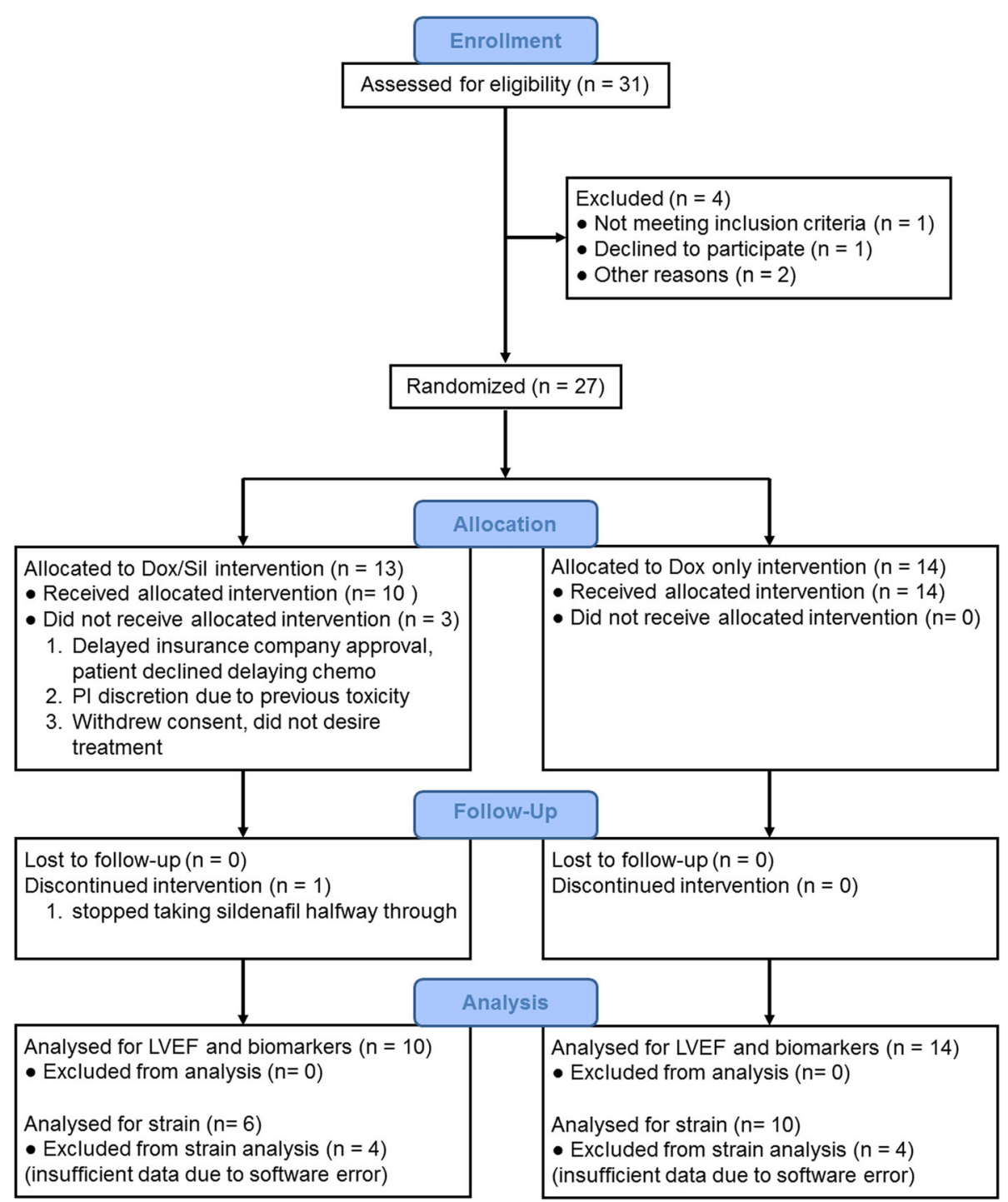

Fig. 1 CONSORT diagram. 31 patients were screened, 27 patients were randomized, and 24 patients were treated with doxorubicin alone or a combination of doxorubicin and sildenafil. Patients were followed up for echocardiograms for up to 4 years after completing chemotherapy 
Table 1 Characteristics of Patients

\begin{tabular}{|c|c|c|}
\hline \multirow[t]{2}{*}{ Characteristic } & $\begin{array}{l}\text { Doxorubicin-Sildenafil } \\
N=10\end{array}$ & $\begin{array}{l}\text { Doxorubicin-only } \\
N=14\end{array}$ \\
\hline & Number (\%) & Number (\%) \\
\hline \multicolumn{3}{|l|}{ Age } \\
\hline Median (Range) & $57(40-77)$ & $50(31-67)$ \\
\hline \multicolumn{3}{|l|}{ Gender } \\
\hline Female & $9(90)$ & $13(93)$ \\
\hline Male & $1(10)$ & $1(7)$ \\
\hline \multicolumn{3}{|l|}{ Race } \\
\hline $\begin{array}{l}\text { American Indian or } \\
\text { Alaska Native }\end{array}$ & $0(0)$ & $1(7)$ \\
\hline Asian & $0(0)$ & $1(7)$ \\
\hline $\begin{array}{l}\text { Black or African } \\
\text { American }\end{array}$ & $2(20)$ & $4(29)$ \\
\hline White & $8(80)$ & $8(57)$ \\
\hline \multicolumn{3}{|l|}{ Tumor Type } \\
\hline Breast Cancer & $8(80)$ & $12(86)$ \\
\hline Ovarian Cancer & $0(0)$ & $1(7)$ \\
\hline Sarcoma & $2(20)$ & $1(7)$ \\
\hline Metastatic Disease & $2(20)$ & $3(21)$ \\
\hline Hypertension & $3(30)$ & $4(29)$ \\
\hline Smoking & $8(80)$ & $3(21)$ \\
\hline Diabetes & $0(0)$ & $0(0)$ \\
\hline Radiation to Heart & $2(20)$ & $5(36)$ \\
\hline Trastuzumab & $2(20)$ & $4(29)$ \\
\hline
\end{tabular}

comparison of LVEF by treatment Arm at the time of the interim analysis was 0.6026 . The LVEF from first to last visit was reduced significantly ( $p$-value $=0.0009$ ), for both Arms. The time effect did not depend on the treatment Arm $(p$-value $=0.8258)$. The study was amended and continued to enroll until a second analysis, when it was determined that it was highly unlikely there would be any cardiac protection identified from sildenafil use during the study. It was determined that sildenafil was safe in combination with doxorubicin.

\section{Cardiac analysis}

Left ventricular ejection fraction decreased over time in the majority of patients (Fig. 2). Most patients in both arms experienced declines in LVEF over time (Doxorubicin/Sildenafil $n=7 / 10$, Doxorubicin-only $n=9 / 14$, total $n=16 / 24)$. However, the T-test result $(p=0.48$, Table 3$)$ indicated no significant difference in change of LVEF between the treatment Arms. Both arms, therefore, were pooled in the analysis of cardiac biomarkers in regard to predictive capabilities for cardiac dysfunction.

For the purposes of identification of patients with cardiac dysfunction resulting from doxorubicin, we defined 2 groups of patients, (LVEF-Group). Patients were allocated
Table 2 Adverse Events

\begin{tabular}{|c|c|c|c|c|}
\hline \multirow[t]{2}{*}{ CTCAE Term } & \multicolumn{4}{|c|}{$\begin{array}{l}\text { \# Patients (\% Patients) } \\
\text { Doxorubicin/Sildenafil N=10 }\end{array}$} \\
\hline & Grade1 & Grade2 & Grade3 & Grade4 \\
\hline Alanine aminotransferase increased & & $1(10)$ & & \\
\hline Alkaline phosphatase increased & $1(10)$ & & & \\
\hline Alopecia & & $3(30)$ & & \\
\hline Anemia & $1(10)$ & $3(30)$ & $1(10)$ & \\
\hline Anorexia & $2(20)$ & $1(10)$ & & \\
\hline Constipation & $2(20)$ & & & \\
\hline Creatinine increased & $1(10)$ & & & \\
\hline Dry eye & $1(10)$ & & & \\
\hline Dysgeusia & $2(20)$ & & & \\
\hline Dyspepsia & $2(20)$ & $2(20)$ & & \\
\hline Edema limbs & $1(10)$ & & & \\
\hline Ejection fraction decreased & & & $3(30)$ & \\
\hline Eye disorders & $3(30)$ & & & \\
\hline Fatigue & $3(30)$ & $3(30)$ & & \\
\hline Fever & & $1(10)$ & & \\
\hline Flushing & $2(20)$ & & & \\
\hline Genital edema & & $1(10)$ & & \\
\hline Headache & $3(30)$ & $1(10)$ & $2(20)$ & \\
\hline Hypocalcemia & $2(20)$ & & & \\
\hline Hypokalemia & $2(20)$ & & & \\
\hline Hypophosphatemia & $1(10)$ & & & \\
\hline Investigations - Other, specify & & $1(10)$ & & \\
\hline Left ventricular systolic dysfunction & & $1(10)$ & & \\
\hline Lip infection & & $1(10)$ & & \\
\hline Lymphocyte count decreased & $2(20)$ & $1(10)$ & $1(10)$ & \\
\hline Mucositis oral & $1(10)$ & $1(10)$ & $1(10)$ & \\
\hline Nail discoloration & $1(10)$ & & & \\
\hline Nausea & $5(50)$ & $2(20)$ & & \\
\hline Peripheral sensory neuropathy & $1(10)$ & $1(10)$ & & \\
\hline Platelet count decreased & $4(40)$ & & & \\
\hline Skin infection & $1(10)$ & & & \\
\hline Vomiting & & & $1(10)$ & \\
\hline
\end{tabular}

into each group for analysis based upon their last follow-up echocardiogram. Cardiac dysfunction patients $(n=3)$ were defined as those having an LVEF of less than $50 \%$ at their final study visit. Retained cardiac function patients $(n=21)$ had LVEF greater than or equal to $50 \%$. Patients were also divided into groups by strain outcome. Ten patients had preserved systolic function as assessed by strain measurements less than $-17 \%$, and 6 patients had abnormal systolic function with strain measurements of greater than or equal to $-17 \%$. Eight patients had incomplete data and could not be categorized by strain outcome. 


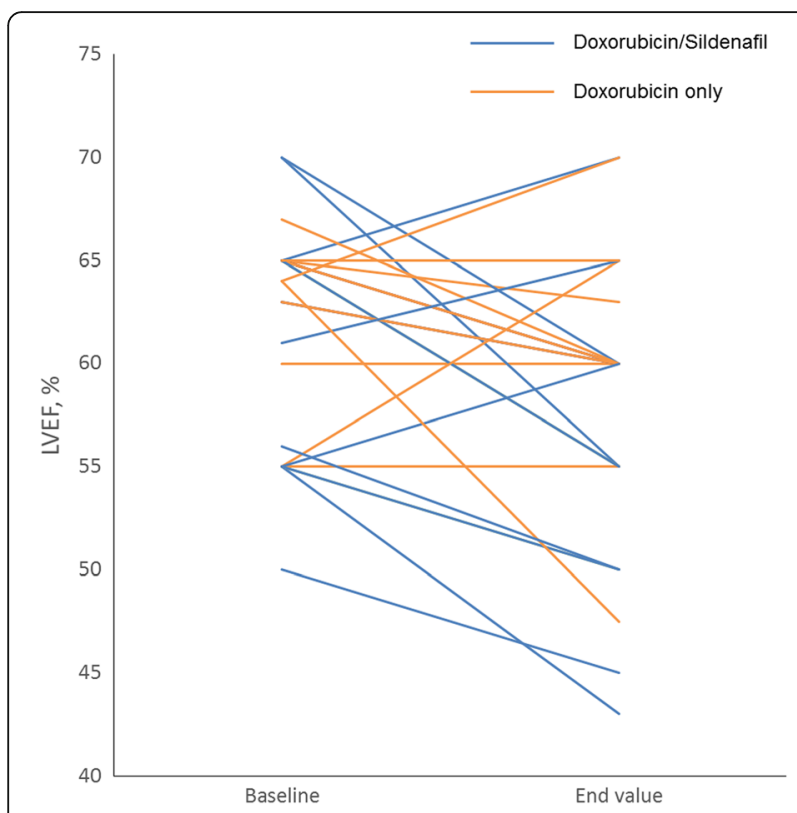

Fig. 2 Changes in LVEF. Of the 24 treated patients, 16 experienced LVEF decline following doxorubicin treatment. 7/10 Patients on doxorubicin-sildenafil and 9/14 patients on doxorubicin only experienced LVEF declines of any grade. LVEF change ranged from a decline of 16.5 percentage points to an increase of 10 percentage points with no significant difference between treatment arms $(p=0.48)$

\section{Cardiac biomarkers: Troponin I}

Serum levels of cTnI were below the lower limit of detection for all patients during the course of the study, and further analysis was not performed.

The changes in hsTnI by cycle categorized by Arm and outcome groups (LVEF, strain) are described in Fig. 3 (a-d). The Arm $\times$ Cycle interaction was explored using a LMM (Fig. 4a). It was observed that with increasing Cycles, the (mean) hsTnI value increased (estimate = $2.60 \mathrm{pg} / \mathrm{mL}, p$-value < 0.0001) for both Arms. No significant difference in (mean) hsTnI value between Arms (doxorubicin-sildenafil; doxorubicin only) was observed (estimate $=0.86 \mathrm{pg} / \mathrm{mL}, p$-value $=0.76)$. Also, the interaction term between Arm and Cycle was not significant (estimate $=-0.13 \mathrm{pg} / \mathrm{mL}, p$-value $=0.90)$. Additionally, no significant difference in mean hsTnI value between the LVEF-Groups (LVEF < 50\% vs. LVEF $\geq 50 \%$ ) was observed (estimate $=1.30, p$-value $=0.63$, data not shown) .

Additionally, to evaluate cardiac injury in the later cycles for the high hsTnI (>10 pg/mL) group vs the low

Table 3 Percentage Point LVEF Change by Arm

\begin{tabular}{lccccc}
\hline Arm & Number of Subjects & Mean & Median & Range & $p$-value \\
\hline $\begin{array}{l}\text { Doxorubicin/ } \\
\text { Sildenafil }\end{array}$ & 10 & -4.9 & -5.5 & 20.0 & 0.4836 \\
Doxorubicin-only & 14 & -2.9 & -3.0 & 26.5 & \\
\hline
\end{tabular}

hsTnI ( $\leq 10 \mathrm{pg} / \mathrm{mL}$ ), the SAS procedure GLIMMIX was used to fit a logistic mixed model on this longitudinal data using two explanatory variables, LVEF-Group and Cycle. The estimated coefficient for Cycle is positive (0.6343) and significant at the 5\% level ( $p$-value $=$ $0.0179)$, implying progressive injury. The area under the curve for this analysis is 0.3056 .

The relationship between the outcome hsTnI and the explanatory factors Strain, Cycle, and Strain $x$ Cycle interaction was explored using a LMM (Fig. $4 \mathrm{~b})$. It was observed that none of these 3 factors were significant ( $p$-values were $0.12,0.12$, and 0.17 , respectively). Strain was not associated with changes in TnI.

\section{Cardiac biomarkers: NT-proBNP}

The changes in NT-proBNP by cycle categorized by Arm and outcome groups (LVEF, strain) are described in Fig. 5(a-c). The relationship between NT-proBNP and the explanatory factors Arm, Cycle, and Arm $\times$ Cycle interaction was explored using a LMM utilizing day 2 data (Fig. 4c). It was observed that with increasing Cycle, the (mean) NT-proBNP did not change significantly $(p$-value $=0.76)$. However, a significant difference in (mean) NT-proBNP value between Arms (Doxorubicin/ Sildenafil vs. Doxorubicin-only) was observed using day 2 data (estimate $=374.44 \mathrm{pg} / \mathrm{mL}, p$-value $=0.034)$. The interaction term between Arm and Cycle was not significant (estimate $=-22.4 \mathrm{pg} / \mathrm{mL}, p$-value $=0.66$ ).

The relationship between the outcome NT-proBNP and the explanatory factors LVEF-Group, Cycle, and LVEF-Group $\times$ Cycle interaction was explored using a LMM. It was observed that none of these 3 factors were statistically significant: LVEF-Group $(p$-value $=0.63)$, Cycle $(p$-value $=0.40)$, and LVEF-Group $\times$ Cycle $(p$-value $=0.64)$.

To assess interaction between Arm and LVEF-Group for predicting NT-proBNP, the explanatory factors Arm, LVEF-Group, Cycle, and the interaction term Arm $\times$ Group were regressed on NT-proBNP using a LMM. We observe that the interaction term Arm $\times$ Group was not significant $(p$-value $=0.63)$; all other factors were also not significant: Arm ( $p$-value $=0.073)$, LVEF-Group ( $p$-value $=0.10)$, and Cycle $(p$-value $=0.48)$. The relationship between NT-proBNP and the explanatory factors Strain, Cycle, and Strain $\times$ Cycle interaction was explored using a LMM. It was observed that none of these 3 factors were significant ( $p$-values were $0.74,0.83,0.58$ respectively).

\section{Cardiac biomarkers: Threshold evaluation}

As part of the peer review process, the question of identification of patients with highest biomarker values potentially having the greatest risk of subsequent cardiac dysfunction was raised. To address this, threshold levels of hsTnI and NT-proBNP were chosen at $10 \mathrm{pg} / \mathrm{mL}$ and $300 \mathrm{pg} / \mathrm{mL}$, 


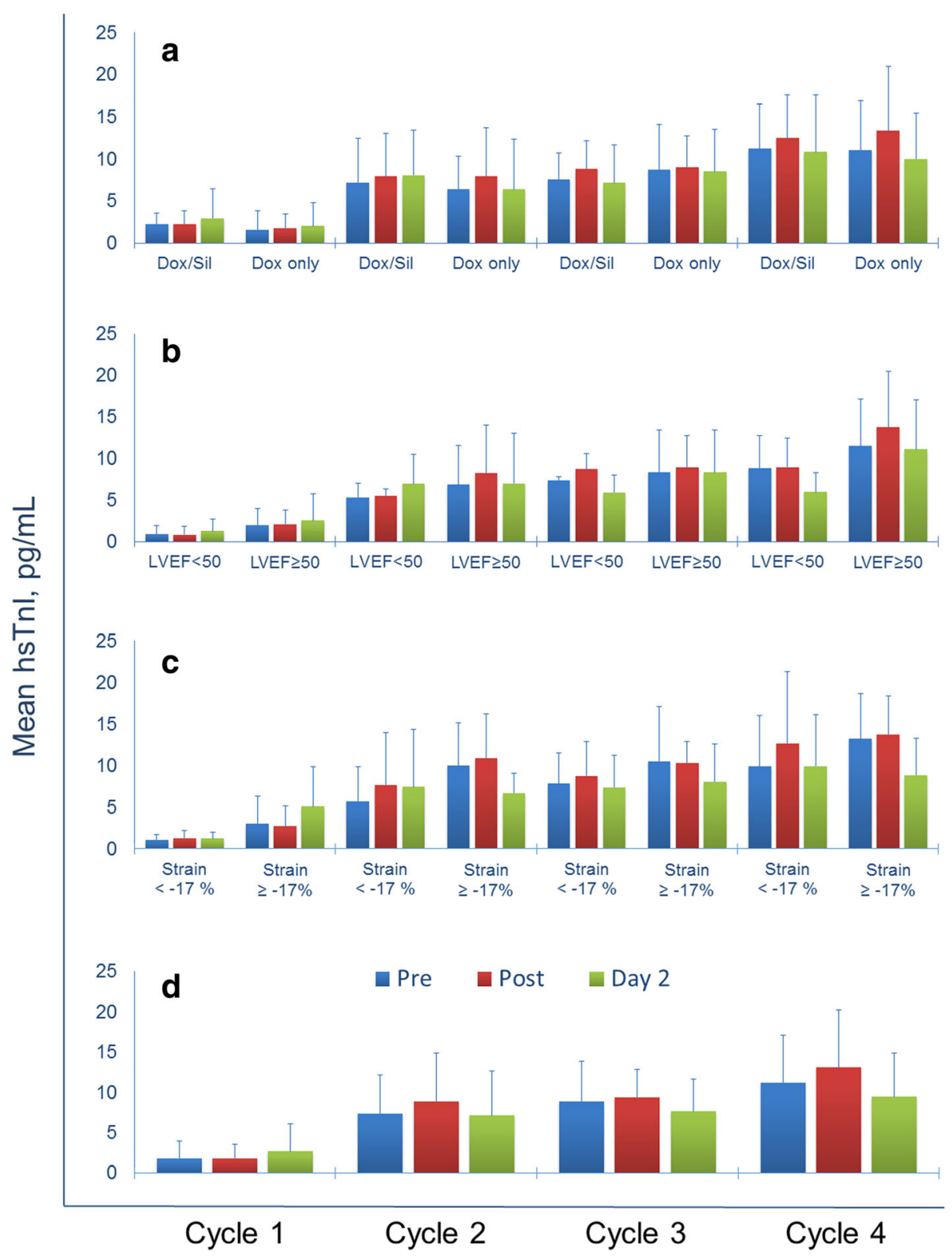

Fig. 3 Mean hsTnl by Cycle. a Mean hsTnl concentrations increased throughout treatment for all outcome groups, indicating cumulative cardiac injury. All values were below what would be clinically detected in a standard troponin screening. Measuring hsTnl during the period of chemotherapy treatment did not predict later heart function decline as measured by either $\mathbf{b}$ LVEF or $\mathbf{c}$ strain. $\mathbf{d}$ There was a statically significant increase between pre- and post- doxorubicin hsTnl values for patients during cycle 2 and cycle 4 ( $p$-value $=0.0029,0.0059$ respectively), an effect not observed with cycle 1 or 3 ( $p$-value $=0.7596,0.2742$ respectively)

respectively. At all 3 measurement time points (pre-treatment, post-treatment, and day 2), the Fisher's exact tests revealed no significant evidence to support that the subjects with LVEF change greater than $10 \%$ were more likely to have high levels of hsTnI or NT-proBNP. The Fisher's exact tests also showed no significant evidence to support that the subjects with strain $\geq-17 \%$ were more likely to have high levels of hsTnI or NT-proBNP. These analyses are presented in $2 \times 2$ tables (Tables 4 and 5).

\section{Discussion}

Anthracyclines are common therapeutic options with curative and palliative intent in many types of cancer. Patients with early stage disease who receive therapy with curative intention can develop delayed systolic cardiac impairment, which diminishes the benefit of anthracyclines and impacts survivorship. Therefore, it is pertinent to develop therapies that can mitigate the cardiotoxicity of treatment and to identify those who are at highest risk of 

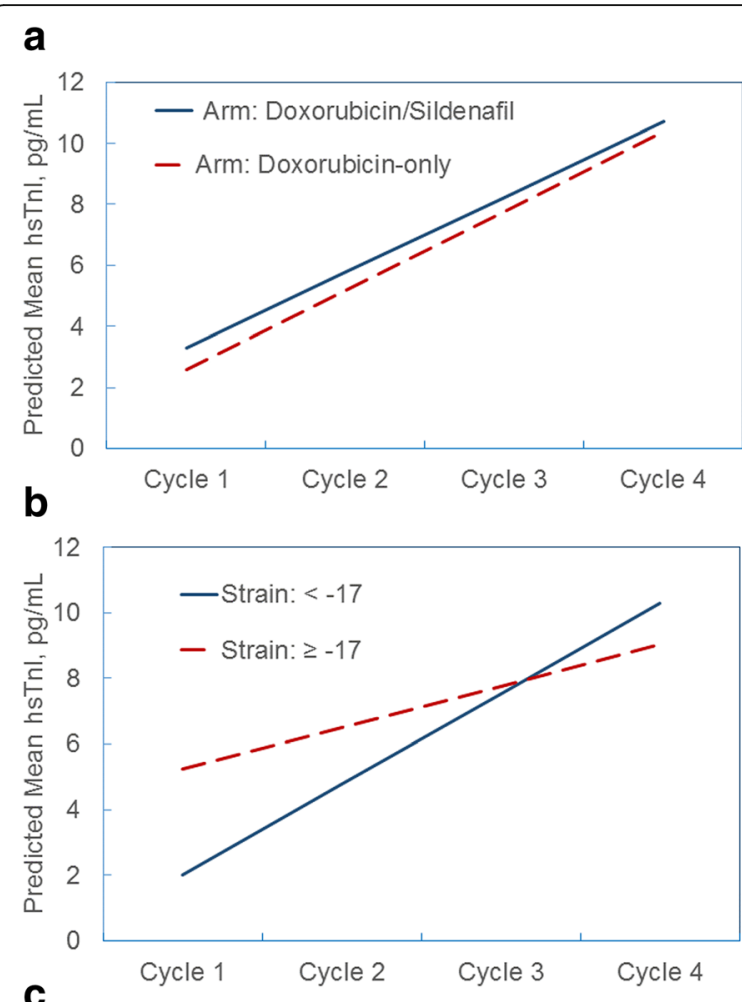

C

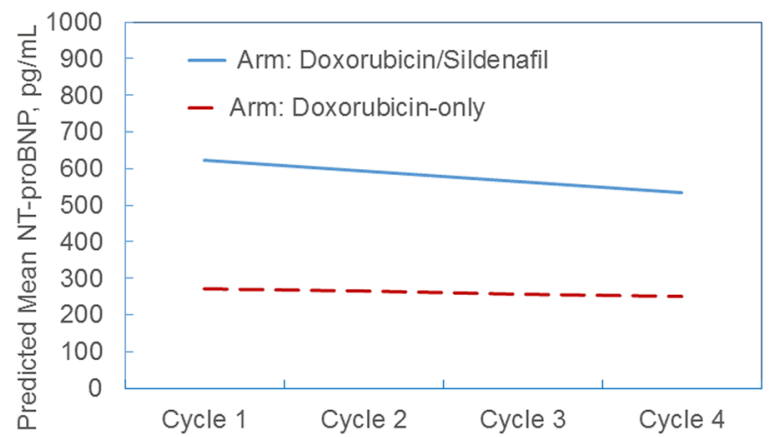

Fig. 4 Linear Mixed Model Predictions of Biomarker Concentrations. $\mathbf{a}$ and $\mathbf{b}$ Linear mixed model analysis of hsTnl concentrations on the day following doxorubicin infusion demonstrated no significant difference between treatment arms or outcome groups. c Linear mixed model analysis of NT-proBNP concentrations on the day following doxorubicin infusion demonstrated significantly higher concentrations in the treatment arm receiving sildenafil

developing subsequent cardiac dysfunction. Dexrazoxane has demonstrated reductions in rates of CHF in patients treated with anthracyclines, and may also be used in selected indications, although use is limited as it increases hematologic toxicity, and the impact on clinical effectiveness has been controversial in some clinical situations. Many cardiologists recommend more intensive management of blood pressure, smoking, diabetes, glucose and cholesterol in patients considered to be a higher risk of late cardiac dysfunction from anthracyclines. Beta blocker and angiotensin receptor blocker therapy can also be employed to help prevent cardiac dysfunction, although their benefit has been inconsistent $[17,18]$.

Our data demonstrated that sildenafil in combination with doxorubicin-based chemotherapy was safe and generally well tolerated. This study did not demonstrate that sildenafil was cardioprotective, although it was underpowered to detect any modest benefit. Most patients (7/ 10) in the sildenafil arm had an LVEF decline, further suggesting against cardiac protection, although the change in LVEF over 4 years of follow-up was modest with an approximately $-5 \%$ decline. Additional long term followup could prove useful, as some patients develop cardiac toxicity much later than the surveillance time-frame of this study [19].

One patient in the doxorubicin-sildenafil arm experienced symptomatic LVEF decline with severe heart failure. Her LVEF measured 50\% at baseline and 69\% at the 6-month follow-up visit, and she was treated with trastuzumab following completion of the course of doxorubicin. However, at her 1-year follow-up visit, this patient's LVEF was $25 \%$, and she was clinically symptomatic. By the time of the 3-year follow-up visit, her LVEF had recovered to $45 \%$. Overall, 5 patients on the study experienced a drop in LVEF to below 50\%. Four of these were in the sildenafil arm, and 2 of those 4 received trastuzumab. Two patients experienced recovery from nadir LVEF to a value $>50 \%$.

\section{Biomarkers}

Previous studies have reported that increases in troponin, including hsTnI, occurring after chemotherapy can identify patients who are likely to develop systolic dysfunction [20]. In contrast, in our study, values of hsTnI did not identify patients at risk of clinically significant LVEF declines $(<50 \%)$. A unique aspect of our study was the serial assessment of hsTnI pre-treatment, immediately post-treatment, and 24$48 \mathrm{~h}$ later. The immediate post-treatment values (during known periods of peak doxorubicin serum concentrations [21]) were increased compared to the pre-treatment values (Fig. 3, panel d), but subsequently decreased over the next $24 \mathrm{~h}$. This is consistent with acute myocardial injury resulting from the doxorubicin. However, serial monitoring (pre-infusion, post-infusion, and day 2) around the day of doxorubicin therapy offered no additional value, as there appeared to be no associated relationship between hsTnI values immediately following doxorubicin administration and subsequent cardiac dysfunction. However, given that the greatest evidence of troponin leak occurred shortly following doxorubicin administration, this may serve as a useful timepoint for future biomarker studies evaluating anthracycline cardiotoxicity. Day 2 hsTnI values did not appear to demonstrate any potential for added utility. In addition, most patients undergoing treatment with doxorubicin in neoadjuvant breast cancer no longer return to the 


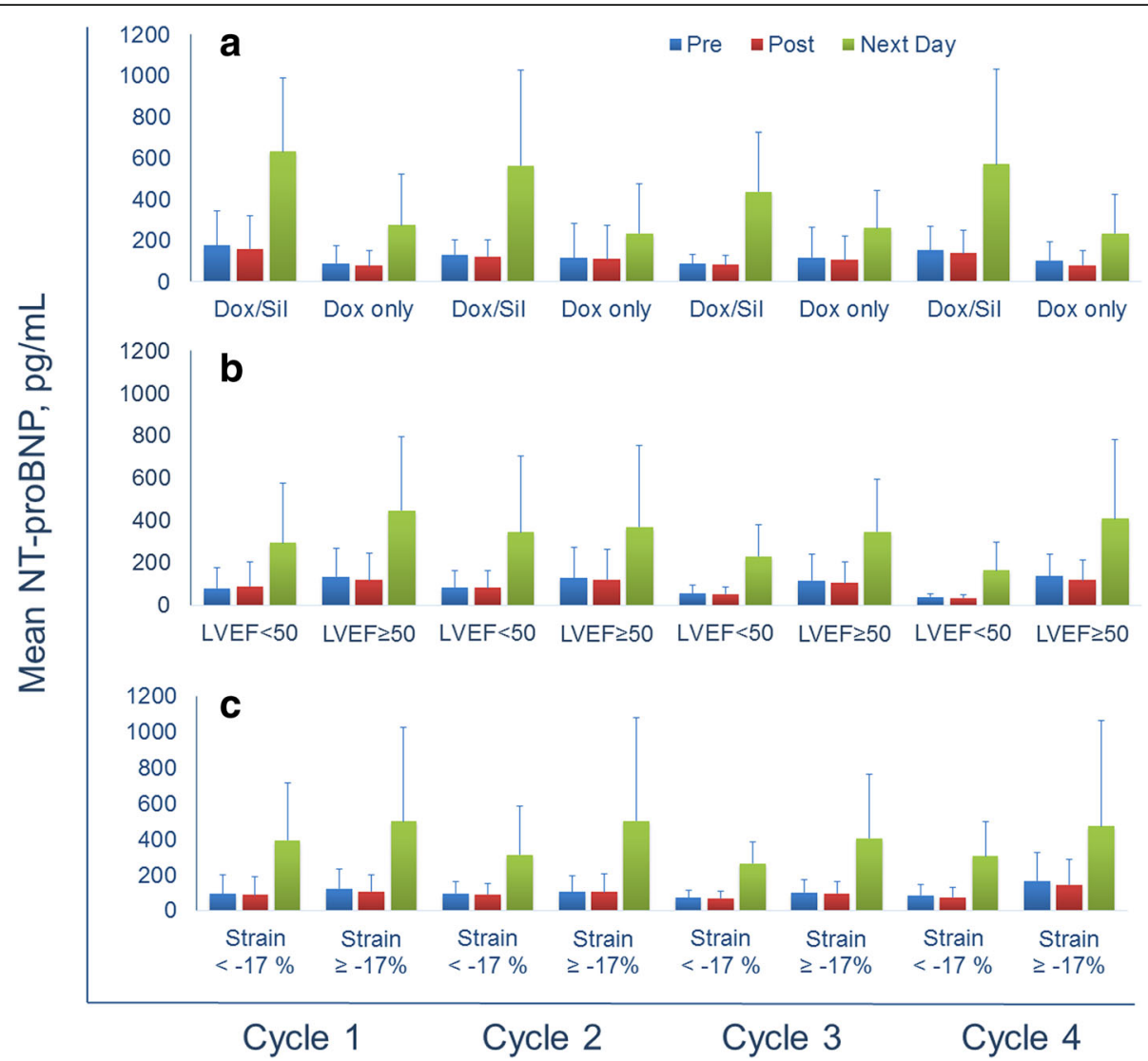

Fig. 5 Mean NT-proBNP by Cycle. All a treatment arms and $\mathbf{b}$ and $\mathbf{c}$ outcome groups experienced temporary spikes in NT-proBNP levels on the day following doxorubicin infusion. However, these levels did not significantly change over the course of chemotherapy

Table 4 Threshold Evaluation of hsTnl

\begin{tabular}{|c|c|c|c|c|c|c|c|}
\hline & & \multicolumn{2}{|l|}{ Pre hsTnl } & \multicolumn{2}{|l|}{ Post hsTnl } & \multicolumn{2}{|l|}{ Day 2 hsTnl } \\
\hline & & $\leq 10 \mathrm{pg} / \mathrm{mL}$ & $>10 \mathrm{pg} / \mathrm{mL}$ & $\leq 10 \mathrm{pg} / \mathrm{mL}$ & $>10 \mathrm{pg} / \mathrm{mL}$ & $\leq 10 \mathrm{pg} / \mathrm{mL}$ & $>10 \mathrm{pg} / \mathrm{mL}$ \\
\hline \multicolumn{8}{|c|}{ LVEF Change } \\
\hline$N$ & $<10 \%(N=17)$ & 10 & 7 & 10 & 7 & 11 & 6 \\
\hline Row \% & & 58.82 & 41.18 & 58.82 & 41.18 & 64.71 & 35.29 \\
\hline $\mathrm{Col} \%$ & & 76.92 & 63.64 & 76.92 & 63.64 & 73.33 & 66.67 \\
\hline $\mathrm{N}$ & $\geq 10 \%(N=7)$ & 3 & 4 & 3 & 4 & 4 & 3 \\
\hline Row \% & & 42.86 & 57.14 & 42.86 & 57.14 & 57.14 & 42.86 \\
\hline $\mathrm{Col} \%$ & & 23.08 & 36.36 & 23.08 & 36.36 & 26.67 & 33.33 \\
\hline & Total & 13 & 11 & 13 & 11 & 15 & 9 \\
\hline \multicolumn{8}{|c|}{ Strain Group } \\
\hline$N$ & $<-17(N=10)$ & 7 & 3 & 6 & 4 & 7 & 3 \\
\hline Row \% & & 70 & 30 & 60 & 40 & 70 & 30 \\
\hline $\mathrm{Col} \%$ & & 87.5 & 37.5 & 85.71 & 44.44 & 70 & 50 \\
\hline $\mathrm{N}$ & $\geq-17(N=6)$ & 1 & 5 & 1 & 5 & 3 & 3 \\
\hline Row \% & & 16.67 & 83.33 & 16.67 & 83.33 & 50 & 50 \\
\hline $\mathrm{Col} \%$ & & 12.5 & 62.5 & 14.29 & 55.56 & 30 & 50 \\
\hline & Total & 8 & 8 & 7 & 9 & 10 & 6 \\
\hline
\end{tabular}


Table 5 Threshold Evaluation of NT-proBNP

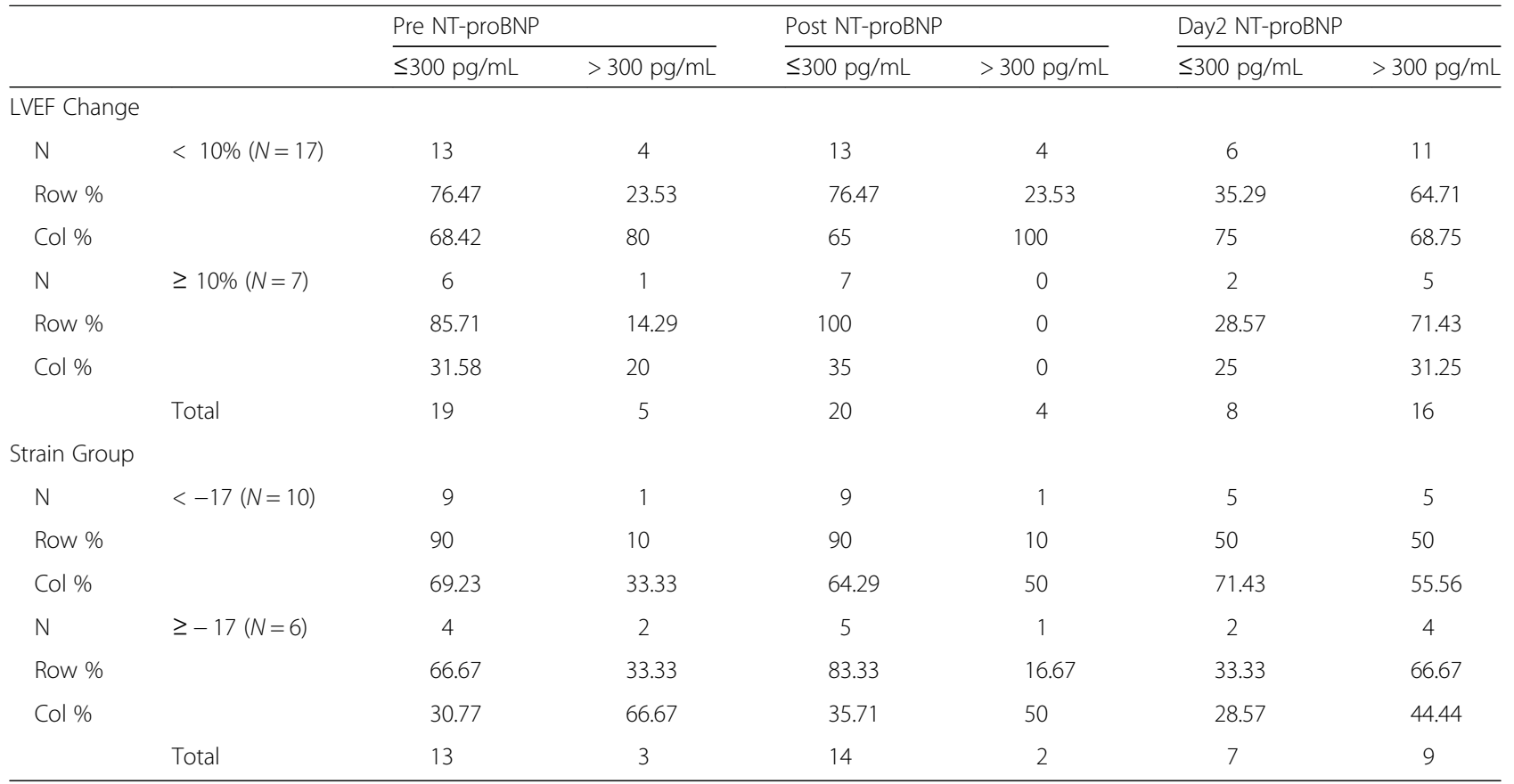

clinic on day 2 after chemotherapy given advances in growth factor support delivery systems, and the results of this study provide no evidence that doing so only for biomarker testing would provide any added prognostic information. In this study hsTnI values increased over time, suggesting subclinical cardiac injury, associated with increased hsTnI release occurring with each doxorubicin dose and progressively higher values with increasing cycle number. Additional studies with more patients and longer follow up could potentially be useful to define whether the increased hsTnI values over time have prognostic value. In larger datasets, tracking new and emerging biomarkers could identify high-risk patients as targets for additional monitoring and intervention.

In our study, NT-proBNP was not a predictive marker. The failure to predict outcomes may be related to the small number of patients who were enrolled, or the lack of significant decline in systolic function. NT-proBNP also failed to identify patients who subsequently developed systolic dysfunction after chemotherapy, a finding reported previously [20]. Elevations in NT-proBNP in the sildenafil arm on day 2 were transient, and thought to be secondary to the vasodilatory effects of high dose sildenafil and were not associated with a long-term negative impact on cardiac function. A similar increase in NT-proBNP with sildenafil treatment was seen in a trial in patients with diastolic heart failure [22].

\section{Study limitations}

This study's sample size is somewhat small for conducting a full-fledged longitudinal analysis using mixed models. However, to understand the effect of time (measured here in cycles of treatment) and the possible significant differences between the Arms, LVEF-Groups, or Strain groups, a longitudinal analysis was necessary.

Additionally, this study enrolled primarily women with breast cancer, most of whom received less cumulative doxorubicin than doses associated with significant cardiac toxicity $\left(300 \mathrm{mg} / \mathrm{m}^{2}\right)$. Therefore, the lack of evidence of a cardioprotective effect cannot be extrapolated to what may be observed in men or patients with other cancer types receiving higher doses of doxorubicin.

\section{Conclusion}

In this pilot study, adding sildenafil to doxorubicin-based chemotherapy did not offer cardiac protection during chemotherapy. Ejection fraction declined over time for most patients (16/24), as expected, following doxorubicin exposure. High sensitivity TnI increased over time, but measuring hsTnI during and immediately after treatment did not identify patients at higher risk for subsequent declines in cardiac function.

\section{Abbreviations}

CHF: Congestive heart failure; CTCAE: Common terminology criteria for adverse events; cTnl: Cardiac troponin l; hsTnl: High-sensitivity cardiac troponin l; LMM: Linear mixed model; LVEF: Left ventricular ejection fraction; NT-proBNP: N-terminal pro-B-type natriuretic peptide; PDE5: Phosphodiesterase 5

\section{Acknowledgements}

Biomarker assays were performed in collaboration with Siemens Healthcare Diagnostics, Newark, DE. 


\section{Funding}

The study was supported, in part, by grants from the National Institutes of Health including P30 CA016059 (Cancer Center Support Grant to the VCU Massey Cancer Center, which also supports the Biostatistics Shared Resource).

\section{Availability of data and materials}

The datasets used and/or analyzed during this study are available from the corresponding author on reasonable request.

\section{Authors' contributions}

AP was a major contributor to the design of the study, treatment of patients, interpreting data, and writing the manuscript. YQ gathered patient data from medical records and was a major contributor in writing the manuscript. MD contributed to interpreting data and critically revising the manuscript. MCK contributed to the design of the study and interpretation of patient data. MK contributed to the design of the biomarker studies and prepared blood samples for biomarker analysis. ES performed echocardiogram analysis. DB and XD performed the statistical analysis and were major contributors to the statistical analysis sections of the manuscript. RCK performed preclinical experiments and contributed to the design of the study. All authors read and approved the final manuscript.

\section{Ethics approval and consent to participate}

This study was reviewed by the Virginia Commonwealth University Institutional Review Board. Informed consent was obtained from all participants.

\section{Consent for publication}

Not applicable; no individual data is reported.

\section{Competing interests}

The authors declare that they have no competing interests.

\section{Publisher's Note}

Springer Nature remains neutral with regard to jurisdictional claims in published maps and institutional affiliations.

\section{Author details}

${ }^{1}$ Massey Cancer Center and Department of Internal Medicine, Division of Hematology-Oncology, Virginia Commonwealth University, Box 980070, Richmond, VA 23298, USA. ²Department of Internal Medicine, Virginia Commonwealth University, Box 980070, Richmond, VA 23298, USA. ${ }^{3}$ Massey Cancer Center, Virginia Commonwealth University, Box 980037, Richmond, VA 23298, USA. ${ }^{4}$ Department of Internal Medicine, Division of Cardiology, Virginia Commonwealth University, Box 980051, Richmond, VA 23298, USA. ${ }^{5}$ Department of Biostatistics, Virginia Commonwealth University, Box 980032 , Richmond, VA 23298, USA.

\section{Received: 21 June 2018 Accepted: 14 August 2018}

Published online: 29 August 2018

\section{References}

1. Sparano JA, Wang M, Martino S, Jones V, Perez EA, Saphner T, Wolff AC, Sledge GW Jr, Wood WC, Davidson NE. Weekly paclitaxel in the adjuvant treatment of breast cancer. N Engl J Med. 2008;358(16):1663-71.

2. Fernandez HF, Sun Z, Yao X, Litzow MR, Luger SM, Paietta EM, Racevskis J, Dewald GW, Ketterling RP, Bennett JM, et al. Anthracycline dose intensification in acute myeloid leukemia. N Engl J Med. 2009; 361(13):1249-59.

3. Coiffier B, Lepage E, Briere J, Herbrecht R, Tilly H, Bouabdallah R, Morel P, Van Den Neste E, Salles G, Gaulard P, et al. CHOP chemotherapy plus rituximab compared with CHOP alone in elderly patients with diffuse largeB-cell lymphoma. N Engl J Med. 2002;346(4):235-42.

4. FDA Statement on Dexrazoxane. In.: U.S. Food and Drug Administration. Drug Saf and Availability; 2011.

5. Tap WD, Jones RL, Van Tine BA, Chmielowski B, Elias AD, Adkins D, Agulnik $M$, Cooney MM, Livingston MB, Pennock $G$, et al. Olaratumab and doxorubicin versus doxorubicin alone for treatment of soft-tissue sarcoma: an open-label phase $1 \mathrm{~b}$ and randomised phase 2 trial. Lancet. 2016; 388(10043):488-97.
6. Pourier MS, Kapusta L, van Gennip A, Bokkerink JP, Loonen J, Bellersen L, Mavinkurve-Groothuis AM. Values of high sensitive troponin $T$ in long-term survivors of childhood cancer treated with anthracyclines. Clin Chim Acta. 2015:441:29-32

7. Blaes AH, Rehman A, Vock DM, Luo X, Menge M, Yee D, Missov E, Duprez D. Utility of high-sensitivity cardiac troponin $T$ in patients receiving anthracycline chemotherapy. Vasc Health Risk Manag. 2015;11:591-4.

8. Cardinale D, Colombo A, Torrisi R, Sandri MT, Civelli M, Salvatici M, Lamantia G, Colombo N, Cortinovis S, Dessanai MA, et al. Trastuzumab-induced cardiotoxicity: clinical and prognostic implications of troponin I evaluation. J Clin Oncol. 2010;28(25):3910-6.

9. Cardinale D, Sandri MT, Colombo A, Colombo N, Boeri M, Lamantia G, Civelli M, Peccatori F, Martinelli G, Fiorentini C, et al. Prognostic value of troponin I in cardiac risk stratification of cancer patients undergoing high-dose chemotherapy. Circulation. 2004;109(22):2749-54.

10. Okumura H, luchi K, Yoshida T, Nakamura S, Takeshima M, Takamatsu H, Ikeno A, Usuda K, Ishikawa T, Ohtake S, et al. Brain natriuretic peptide is a predictor of anthracycline-induced cardiotoxicity. Acta Haematol. 2000; 104(4):158-63.

11. Di X, Gennings C, Bear HD, Graham LJ, Sheth CM, White KL Jr, Gewirtz DA Influence of the phosphodiesterase-5 inhibitor, sildenafil, on sensitivity to chemotherapy in breast tumor cells. Breast Cancer Res Treat. 2010;124(2): 349-60.

12. Fisher PW, Salloum F, Das A, Hyder H, Kukreja RC. Phosphodiesterase-5 inhibition with sildenafil attenuates cardiomyocyte apoptosis and left ventricular dysfunction in a chronic model of doxorubicin cardiotoxicity. Circulation. 2005;111(13):1601-10.

13. Das A, Durrant D, Mitchell C, Mayton E, Hoke NN, Salloum FN, Park MA, Qureshi I, Lee R, Dent P, et al. Sildenafil increases chemotherapeutic efficacy of doxorubicin in prostate cancer and ameliorates cardiac dysfunction. Proc Natl Acad Sci U S A. 2010;107(42):18202-7.

14. Koka S, Das A, Zhu SG, Durrant D, Xi L, Kukreja RC. Long-acting phosphodiesterase-5 inhibitor tadalafil attenuates doxorubicin-induced cardiomyopathy without interfering with chemotherapeutic effect. J Pharmacol Exp Ther. 2010;334(3):1023-30.

15. Citron ML, Berry DA, Cirrincione C, Hudis C, Winer EP, Gradishar WJ, Davidson NE, Martino S, Livingston R, Ingle JN, et al. Randomized trial of dose-dense versus conventionally scheduled and sequential versus concurrent combination chemotherapy as postoperative adjuvant treatment of node-positive primary breast cancer: first report of intergroup trial C9741/Cancer and leukemia group B trial 9741. J Clin Oncol. 2003;21(8): 1431-9.

16. Chen J, Sarkar SK. A Bayesian determination of threshold for identifying differentially expressed genes in microarray experiments. Stat Med. 2006; 25(18):3174-89.

17. Avila MS, Ayub-Ferreira SM, de Barros Wanderley MR Jr, das Dores Cruz F, Goncalves Brandao SM, Rigaud VOC, Higuchi-Dos-Santos MH, Hajjar LA, Kalil Filho R, Hoff PM, et al. Carvedilol for prevention of chemotherapy-related cardiotoxicity: The CECCY Trial. J Am Coll Cardiol. 2018;71(20):2281-90.

18. Bosch X, Rovira M, Sitges M, Domenech A, Ortiz-Perez JT, de Caralt TM, Morales-Ruiz M, Perea RJ, Monzo M, Esteve J. Enalapril and carvedilol for preventing chemotherapy-induced left ventricular systolic dysfunction in patients with malignant hemopathies: the OVERCOME trial (preventiOn of left ventricular dysfunction with Enalapril and caRvedilol in patients submitted to intensive ChemOtherapy for the treatment of malignant hEmopathies). J Am Coll Cardiol. 2013;61(23):2355-62.

19. Du XL, Xia R, Liu CC, Cormier JN, Xing Y, Hardy D, Chan W, Burau K. Cardiac toxicity associated with anthracycline-containing chemotherapy in older women with breast cancer. Cancer. 2009;115(22):5296-308.

20. Putt M, Hahn VS, Januzzi JL, Sawaya H, Sebag IA, Plana JC, Picard MH, Carver $J R$, Halpern EF, Kuter I, et al. Longitudinal changes in multiple biomarkers are associated with cardiotoxicity in breast Cancer patients treated with doxorubicin, Taxanes, and Trastuzumab. Clin Chem. 2015;61(9):1164-72.

21. Benjamin RS, Riggs CE Jr, Bachur NR. Plasma pharmacokinetics of adriamycin and its metabolites in humans with normal hepatic and renal function. Cancer Res. 1977;37(5):1416-20.

22. Redfield MM, Chen HH, Borlaug BA, Semigran MJ, Lee KL, Lewis G, LeWinter MM, Rouleau JL, Bull DA, Mann DL, et al. Effect of phosphodiesterase-5 inhibition on exercise capacity and clinical status in heart failure with preserved ejection fraction: a randomized clinical trial. JAMA. 2013;309(12):1268-77. 